\title{
Computational fluid dynamics simulation of the right subclavian artery cannulation
}

Satoshi Numata, MD, PhD, Keiichi Itatani, MD, PhD, Hidetake Kawajiri, MD, PhD, Sachiko Yamazaki, MD, PhD, Keiichi Kanda, MD, PhD, and Hitoshi Yaku, MD, PhD

\section{ABSTRACT}

Objective: The purpose of this study was to evaluate the efficacy of right subclavian artery cannulation using computational fluid dynamics.

Methods: Patient-specific models of the aortic arch were made with 4 patterns (1: normal aorta, 2: ascending aorta aneurysm, 3: distal arch aneurysm, 4: bovine arch) based on the computed tomography images. Right subclavian artery and ascending aorta cannulation models were created to simulate the physiologic pulsatile flow. Perfusion flow through the arterial cannula was set to $2.50 \mathrm{~L} / \mathrm{min}$ (50\% flow), $3.75 \mathrm{~L} / \mathrm{min}$ (75\% flow), and $5.0 \mathrm{~L} / \mathrm{min}(100 \%)$, respectively, and a 3-dimensional movie was made of 1 cardiac cycle to evaluate the blood flow.

Results: In both $50 \%$ and $75 \%$ flow simulation with right subclavian artery cannulation, the blood streamline from the right subclavian artery produced retrograde flow of the brachiocephalic artery and antegrade flow of the right common carotid artery throughout the cardiac cycle in all cases. Right subclavian artery flow deflected ascending aorta flow to the descending aorta. Left-side supraaortic branches were perfused by blood flow from both the right subclavian artery cannula and the aortic valve. The ascending aortic cannulation model showed that blood flow from the aortic valve reached all 3 supra-aortic vessels in systole.

Conclusions: Right subclavian artery cannulation was cerebroprotective, especially on the right side. (J Thorac Cardiovasc Surg 2017;154:480-7)



Computer simulation of rSCA cannulation of 4 different anatomies.

\section{Central Message}

rSCA cannulation was simulated by CFD, which showed that cannulation produced a protective effect on the brain, especially on the right side.

\section{Perspective}

The brain protection effect of rSCA cannulation is well known; however, the mechanism is not well understood. Blood flow streamline can be visualized by computer stimulation, and now we can understand how rSCA cannulation influences systemic blood circulation. Our results may change the arterial cannulation strategy significantly.

See Editorial Commentary page 488.
Preoperative and intraoperative evaluations of the aorta with computed tomography, transesophageal echocardiography, and epiaortic ultrasound are mandatory in patients with systemic atherosclerosis. To prevent embolization during cardiopulmonary bypass, it is essential to choose the best arterial cannulation site. The right subclavian artery (rSCA) is easy to expose, and it is sufficient for arterial cannulation. Acute aortic dissection rarely extends to the rSCA. $^{1,2}$ Many reports have shown the positive clinical impacts of rSCA cannulation..$^{3-7}$ However, the mechanism of how perfusion of the rSCA prevents cerebral embolism has not been established. Blood flow analysis by computational fluid dynamics (CFD) has become more popular. CFD can evaluate the flow streamline, flow velocity, wall shear stress, helicity index, and other fluid dynamic parameters. ${ }^{8-10}$ CFD also enables visualization of blood flow. We describe our experience with visualizing blood flow from the aortic root to the descending aorta with rSCA cannulation and ascending aorta (AscAo) cannulation during cardiopulmonary bypass.

From the Department of Cardiovascular Surgery, Kyoto Prefectural University of Medicine, Kyoto, Japan.

Read at the 96th Annual Meeting of The American Association for Thoracic Surgery, Baltimore, Maryland, May 14-18, 2016.

Received for publication May 18, 2016; revisions received Jan 19, 2017; accepted for publication Feb 14, 2017; available ahead of print May 5, 2017.

Address for reprints: Satoshi Numata, MD, PhD, Department of Cardiovascular Surgery, Kyoto Prefectural University of Medicine, 465 Kajiicho Kamigyo, Kyoto, Japan 6028566 (E-mail: snumat@yahoo.co.jp).

$0022-5223 / \$ 36.00$

Copyright (c) 2017 by The American Association for Thoracic Surgery

http://dx.doi.org/10.1016/j.jtcvs.2017.02.073

Scanning this QR code will take you to a supplemental video. To view the AATS 2016 Webcast, see the URL next to the webcast thumbnail.

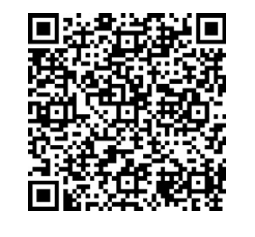




\section{Abbreviations and Acronyms \\ AscAo $=$ ascending aorta \\ $\mathrm{CFD}=$ computational fluid dynamics \\ MRI = magnetic resonance imaging \\ rSCA $=$ right subclavian artery \\ $3 \mathrm{D}=3$-dimensional}

\section{MATERIALS AND METHODS}

\section{Study Design for Computational Analysis}

We evaluated rSCA and AscAo cannulation by using CFD in 4 patients. Perfusion flow through the rSCA or AscAo cannula was set to $2.50 \mathrm{~L} / \mathrm{min}$ ( $50 \%$ flow), $3.75 \mathrm{~L} / \mathrm{min}$ ( $75 \%$ flow), and $5.0 \mathrm{~L} / \mathrm{min}$ $(100 \%)$ at a constant, steady flow. In addition, a 3-dimensional (3D) movie was made for 1 cardiac cycle to evaluate the streamline of the blood flow and velocity.

\section{Patient Profiles for Computational Analysis}

We chose 4 patients with different aortic arch pathologies: patient 1, with a normal size aortic arch; patient 2 , with an ascending aortic aneurysm with a unicuspid aortic valve; patient 3, with a distal arch aneurysm; and patient 4, with a bovine aortic arch with a normal-sized aorta. Patient 1 was a 76year-old man who underwent coronary artery bypass grafting for stable angina. Preoperatively, he underwent evaluation of the thoracic aorta, which was normal in size; therefore, we used his data for a control. Patient 2 was a 72-year-old man who had severe aortic valve stenosis and elongation of the AscAo with dilatation (diameter, $45 \mathrm{~mm}$ ). AscAo and aortic valve replacement were performed. Patient 3 was an 80-year-old man who underwent total arch replacement for a distal arch aneurysm (maximum diameter, $60 \mathrm{~mm}$ ). Patient 4 was a 79 -year-old woman who underwent coronary artery bypass grafting. The postoperative computed tomography scan showed a bovine aortic arch, and the right and left common carotid arteries arose from the common trunk. The study was approved by the institutional review board of Kyoto Prefectural University of Medicine, and written informed consent was obtained from all participating patients.

\section{Computational Simulation}

The methods of our computational analysis have been described. ${ }^{8,9}$ Data for the analyses were acquired by thin-slice, early-phase, enhanced multidetector-row computed tomography imaging. Image data in a Digital Imaging and Communications in Medicine format were transferred into 3D patient-specific geometries by using Osirix, a medical open source imaging software (Osirix Foundation, Geneva, Switzerland). The images were modified by 3D-Coat, a 3D computer graphics software program (PIGWAY, Kiev, Ukraine). For rSCA cannulation, end-to-side anastomosis between the rSCA and an 8-mm prosthetic graft was simulated. For AscAo cannulation, the insertion of an 8-mm AscAo cannula into the AscAo was simulated. Computational meshes were created with ANSYS-ICEM CFD 16.0 (ANSYS, Japan, Tokyo, Japan). More than 2,000,000 cells with tetrahedral meshes and 3 boundary-fitted prism layers were generated at the boundaries of the rSCA and AscAo.

\section{Boundary Conditions}

To simulate the flow around the valve leaflets, inlet boundaries for the AscAo and cannulation site were extruded to 5 times their diameters to develop the velocity profiles. The inlet boundary conditions in the AscAo were set as the mass flow boundary conditions with a pulsatile wave, and those in the subclavian and AscAo perfusion cannulas were set as the constant steady flow rate conditions. Cardiac outputs were set at $5.0 \mathrm{~L} / \mathrm{min}$. Pressure waveforms in 1 case during cardiopulmonary bypass of $50 \%$ and $75 \%$ pump flow were measured. Then, on the basis of the measured pressure waveform, the boundary pressure conditions were derived to realize reflection wave and vascular inertance, and were given at the extended boundary mesh. The outlet boundaries for the supra-aortic branches, descending aorta, and both coronary arteries were extended to 50 times the diameter of each vessel to obtain a stable flow split to the branches, based on our previous validation study on aortic CFD modeling. ${ }^{10}$ The outlet boundary conditions were used as the pressure boundary conditions, which represented the external forces from outside of the analysis domain. One of the main external forces from the peripheral tissue is a reflection wave; thus, to determine the reflection, the following formula was used:

$$
\mathrm{P}_{\text {measured }}-\mathrm{Z}_{0} \mathrm{Q}_{\text {inlet }}
$$

where $P_{\text {measured }}$ is the measured pressure wave, $\mathrm{Q}_{\text {inlet }}$ is the total inlet flow, and $\mathrm{Z}_{0}$ is the characteristic impedance of the aorta. To determine the inertial properties of the vessel wall, we set the inertial term with inductance $L$ to maintain the intravessel pressure with the flow change as follows:

$$
-\mathrm{L} \frac{\mathrm{dQ}_{\text {inlet }}}{\mathrm{dt}}
$$

In addition, the aortic flow is typically regulated by the autonomous system, such as the vasovagal reflex, to prevent flow voids from the neck vessels or excessive current to the lower half body, which can occur when the flow decreases in the late systolic phase. To simulate vascular bed constriction or dilatation according to the abnormal flow split, we added peripheral tonus regulation term for flow split modulation as follows:

$$
\mathrm{R}_{\text {peripheral }} \times \mathrm{H}\left(\mathrm{Q}_{\text {descending }}-\mathrm{Q}_{\text {inlet }}\right)
$$

where $\mathrm{R}_{\text {peripheral }}$ is the peripheral arterial resistance (constant) and $\mathrm{xH}$ is the Heaviside function. Outlet boundaries in the coronary arteries were set as the mass flow boundary conditions with pulsatility, and $2.5 \%$ of the total aortic flow was released into the right and left coronary arteries. The vessel walls, including the extended boundary walls, were considered rigid (Figure 1).

\section{Turbulent Pulsatile Flow Simulations}

The finite volume solver package ANSYS-FLUENT 15.0 or 16.0 (ANSYS, Japan) was used to solve the Navier-Stokes equation of incompressible transient Newtonian fluid. The blood properties were set as follows: $1060 \mathrm{~kg} / \mathrm{m}^{3}$ for density and $0.004 \mathrm{~kg} / \mathrm{m} / \mathrm{s}$ for viscosity. Because the Reynolds number was approximately 4000 in the peak systolic phase, we analyzed the turbulent flow simulation by using Renormalization Group k- $\varepsilon$ models. In the transient flow simulation, each time step was set to $10^{-5}$ seconds to reduce the Courant number to the sufficient level. The convergence criteria were set to $10^{-5}$ times the residual for all degrees of the parameters at each time step.

\section{RESULTS \\ Right Subclavian Artery Cannulation}

We classified flow distribution of the supra-aortic branches into 4 different subsets: grade 4 , totally perfused by cannulation flow; grade 3 , greater than $50 \%$ perfused by cannulation flow; grade 2 , partially perfused by cannulation flow; and grade 1, no perfusion from cannulation flow (Table 1).

In all rSCA cannulation models, the right common carotid artery was a grade 4 in systole and diastole with $50 \%$ and $75 \%$ flow (Table 1), indicating that the right common carotid artery was perfused by blood flow from the rSCA cannula during the entire cardiac cycle regardless of cardiopulmonary bypass flow (Figure 2, Video 1). 


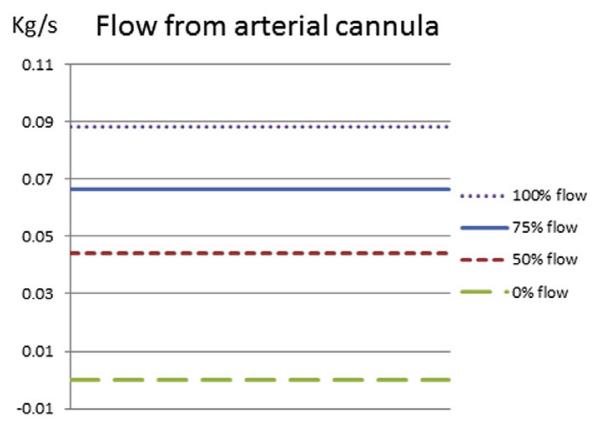

$\mathrm{Kg} / \mathrm{s}$ Flow from aortic valve
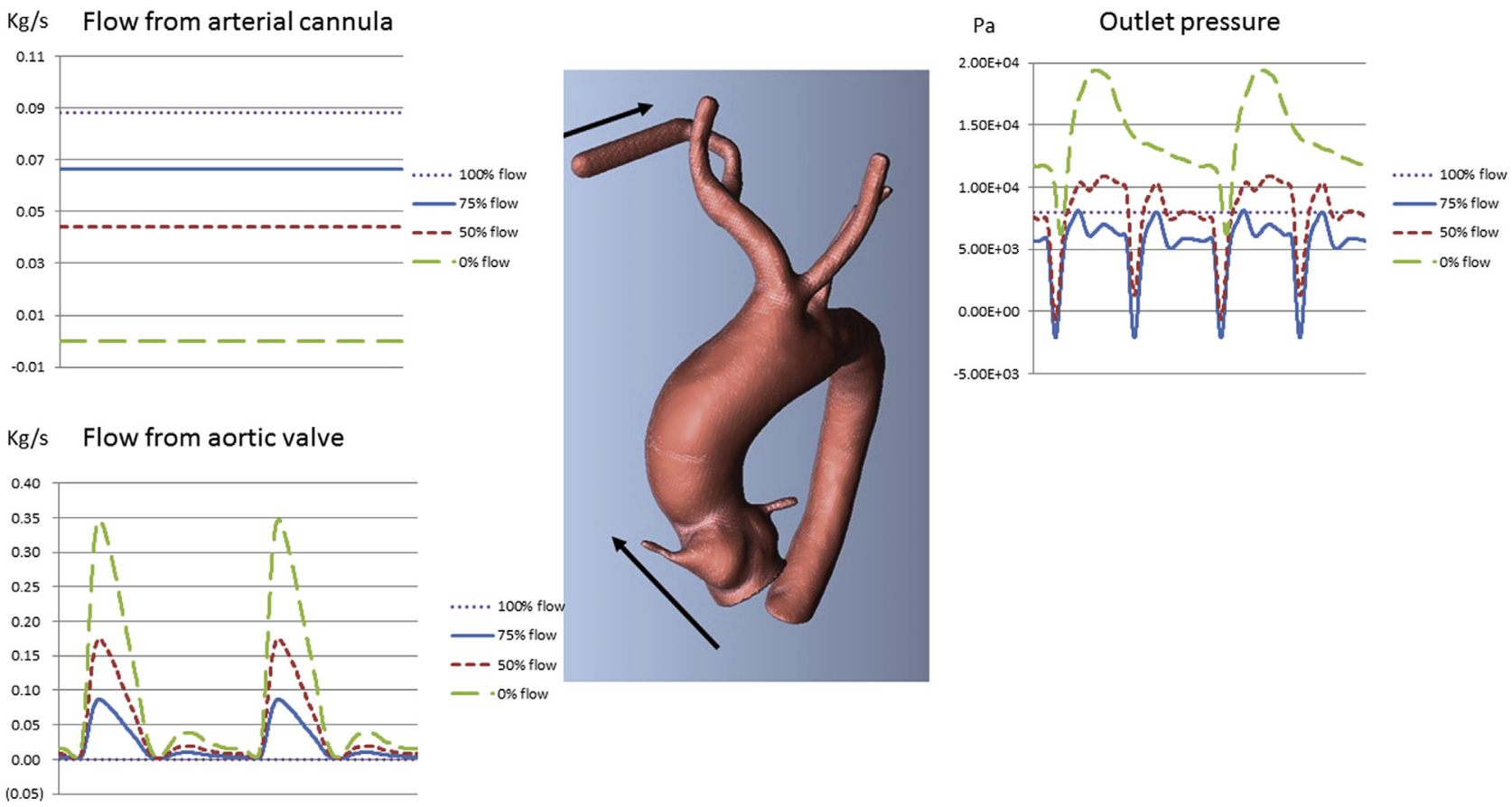

FIGURE 1. Boundary conditions of CFD.

In regard to the left common carotid artery, there was a trend in that most blood flow was supplied from the rSCA cannula in diastole in all models; thus, all cases had a grade 3 or 4 flow (Table 1). In systole, the flow distribution was different for each case. In patients 1 and 2, the left common

TABLE 1. Flow distribution of right subclavian artery and ascending aorta cannulation

\begin{tabular}{|c|c|c|c|c|c|c|c|c|}
\hline & \multicolumn{2}{|c|}{ Patient 1} & \multicolumn{2}{|c|}{ Patient 2} & \multicolumn{2}{|c|}{ Patient 3} & \multicolumn{2}{|c|}{ Patient 4} \\
\hline & $\mathbf{5 0} \%$ & $75 \%$ & $50 \%$ & $\mathbf{7 5} \%$ & $50 \%$ & $75 \%$ & $\mathbf{5 0} \%$ & $\mathbf{7 5} \%$ \\
\hline \multicolumn{9}{|l|}{$\mathrm{rSCA}$} \\
\hline \multicolumn{9}{|l|}{ Systole } \\
\hline RCCA & 4 & 4 & 4 & 4 & 4 & 4 & 4 & 4 \\
\hline LCCA & 4 & 4 & 3 & 3 & 1 & 1 & 3 & 2 \\
\hline LSCA & 1 & 1 & 1 & 3 & 2 & 1 & 1 & 3 \\
\hline \multicolumn{9}{|l|}{ Diastole } \\
\hline RCCA & 4 & 4 & 4 & 4 & 4 & 4 & 4 & 4 \\
\hline LCCA & 4 & 3 & 4 & 3 & 3 & 3 & 3 & 4 \\
\hline LSCA & 2 & 2 & 4 & 3 & 4 & 2 & 3 & 3 \\
\hline \multicolumn{9}{|l|}{ AscAo } \\
\hline \multicolumn{9}{|l|}{ Systole } \\
\hline RCCA & 1 & 2 & 3 & 2 & 2 & 2 & 2 & 3 \\
\hline LCCA & 1 & 1 & 3 & 3 & 4 & 3 & 2 & 2 \\
\hline LSCA & 1 & 2 & 3 & 2 & 4 & 4 & 1 & 2 \\
\hline \multicolumn{9}{|l|}{ Diastole } \\
\hline RCCA & 3 & 3 & 3 & 3 & 3 & 4 & 4 & 4 \\
\hline LCCA & 4 & 4 & 4 & 4 & 4 & 4 & 4 & 4 \\
\hline LSCA & 3 & 3 & 3 & 4 & 2 & 3 & 4 & 4 \\
\hline
\end{tabular}

$1=$ no perfusion from cannulation flow; $2=$ partially perfused by cannulation flow; $3=$ more than $50 \%$ perfused by cannulation flow; $4=$ totally perfused by cannulation flow. $r S C A$, Right subclavian artery; $R C C A$, right common carotid artery; $L C C A$, left common carotid artery; $L S C A$, left subclavian artery; AscAo, ascending aorta. carotid artery was a grade 3 or 4 flow in systole and diastole. In patient 4 , the flow of the rSCA was still dominant, except in systole with $75 \%$ flow. However, in patient 3 , the flow of the rSCA did not reach the left common carotid artery with $50 \%$ and $75 \%$ flow in systole.

Generally, in the left subclavian artery, blood flow from the native aortic valve became more dominant in all cases. Especially in the systolic phase at 50\% flow, patients 1,2, and 4 had a grade 1 flow and patient 3 had a grade 2 flow. With $75 \%$ flow, the flow of the rSCA was increased to grade 3 in patients 2 and 4 . However, patients 1 and 3 had a grade 1 flow. Overall, during most cardiac cycles, especially the systolic phase, the left subclavian artery was likely to be perfused by blood from the aortic valve.

\section{Ascending Aorta Cannulation}

Generally, in systole, the flow of the AscAo and aortic valve streamed into all 3 vessels in all models. For the right common carotid artery, there was a trend in that the blood flow streamed into the right common carotid artery almost equally in systole and diastole. In diastole, flow from the AscAo cannula became more dominant in all cases. In patients 3 and 4, the right common carotid artery had a grade 4 flow in diastole with $75 \%$ flow. In the left common carotid artery, flow from the AscAo was more dominant than from the other supra-aortic vessels. In diastole with $50 \%$ and $75 \%$ flow, the flow distribution was a grade 4 in all cases. In the diastolic phase, the left common carotid artery was totally perfused by blood flow from the AscAo cannula. 


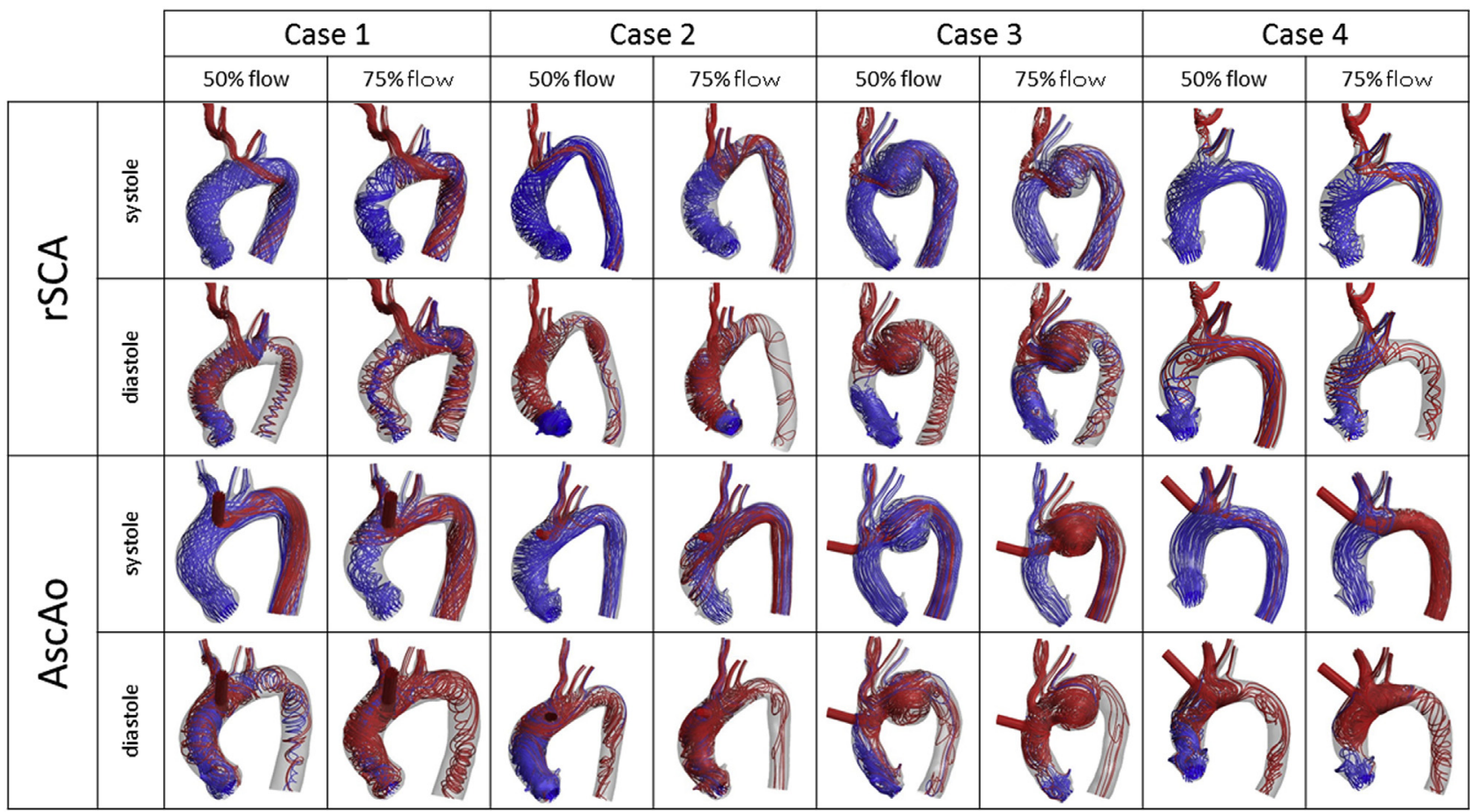

FIGURE 2. CFD simulation results of rSCA cannulation and ascending aortic cannulation. Red line indicates blood streamline from arterial cannula. Blue line indicates blood flow from aortic valve. AscAo, Ascending aorta; $r S C A$, right subclavian artery.

In the left subclavian artery, there was no uniform trend. In patients 1,2, and 4, flow of the AscAo became dominant in diastole.



VIDEO 1. CFD results. 3D movie of 1 cardiac cycle of rSCA cannulation and AscAo cannulation. Video available at: http://www.jtcvsonline.org/ article/S0022-5223(17)30680-3/addons.

\section{Flow Velocity}

A comparison of the flow velocity inside the aorta and branches at each cannulation site is shown in Figure 3. With $50 \%$ flow, there were no obvious differences between the groups. As the pump flow increased to $75 \%$, flow velocity of the supra-aortic vessels was higher with rSCA cannulation than with AscAo cannulation.

\section{$100 \%$ Flow}

The results of $100 \%$ flow simulations of both rSCA and AscAo cannulation are shown in Figure 3. In rSCA series, flow velocity increased inside aorta near the orifice of the brachiocephalic artery, which was caused by the jet flow from rSCA cannulation. By AscAo cannulation, the flow velocity of supra-aortic vessels was decreased compared with the $50 \%$ and $75 \%$ models.

\section{DISCUSSION}

A lower neurologic deficit rate after total arch replacement has been reported with rSCA cannulation. ${ }^{3,4}$ Svensson and colleagues ${ }^{5}$ reported that the rSCA inflow plus the graft reduced the rate of stroke after surgery when hypothermic circulatory arrest was used. Etz and colleagues $^{7}$ compared the surgical results of the aortic root and AscAo surgery by different aortic cannulation sites. They reported a lower incidence of mortality and stroke with rSCA cannulation. A lower morbidity is associated with using subclavian artery cannulation for 


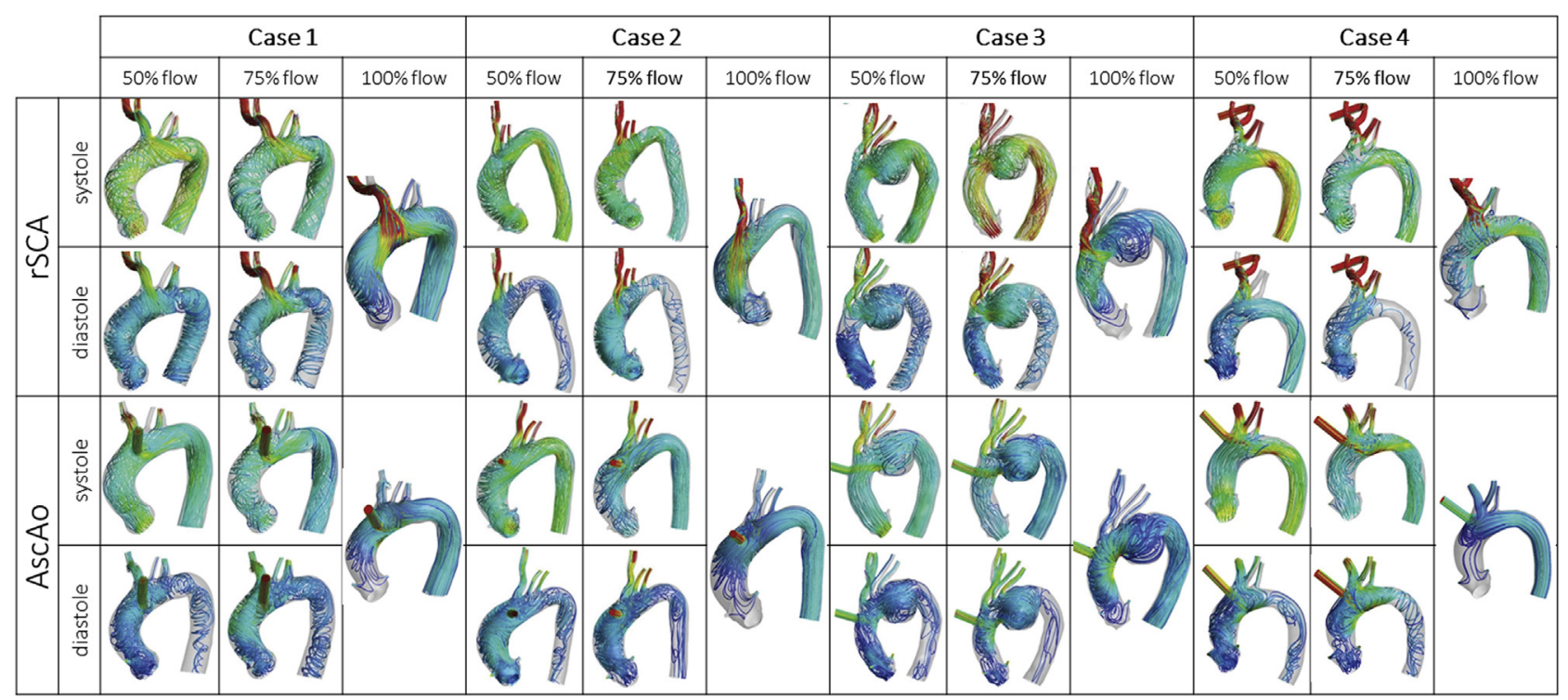

FIGURE 3. CFD simulation results of rSCA cannulation and ascending aortic cannulation. Flow velocity. AscAo, Ascending aorta; $r S C A$, right subclavian artery.

aortic surgery and cases with a conventional coronary or valve with severe atherosclerotic AscAo. ${ }^{6}$

On the other hand, problems with rSCA cannulation have been reported. For example, direct cannulation of the rSCA cannulation can lead to an obstruction of the vertebral artery's orifice by an arterial cannula. ${ }^{11}$ It is not uncommon for us to encounter the aortic arch anomaly, such as aberrant subclavian artery and bovine aortic arch. The existence of these anomalies could influence the strategy of brain protection with rSCA cannulation. Therefore, surgeons should be aware of an aortic anomaly at the time of cannulating the rSCA, especially with acute type A aortic dissection surgery. ${ }^{12-14}$

Although several authors have described the positive impacts of rSCA cannulation, the mechanism of cerebral protection is still uncertain. Some have tried to evaluate the hemodynamics of the aortic arch when subclavian artery cannulation is used. Hedayati and colleagues ${ }^{15}$ performed an experimental study on right axillary artery cannulation. They used cardiopulmonary bypass by using the right axillary artery or AscAo cannulation in a dog model. Microspheres were injected into the AscAo, and they evaluated the distribution of microspheres in the brain, kidney, and skeletal muscles. They concluded that right axillary artery cannulation has a cerebroprotective effect, because axillary artery cannulation resulted in $73 \%$ fewer microspheres in the right brain and $40 \%$ fewer microspheres in the left brain than AscAo cannulation. Of note, the right side of the brain is more effectively protected by microspheres than the left side. In an experimental study by Minakawa and colleagues, ${ }^{16}$ a transparent glass model of a normal aortic arch was used to observe flow and wall shear stress. They concluded that flow in the aortic arch and the AscAo was unexpectedly rapid, especially in the neck of the arch vessel and lesser curvature of the arch.

These previous experimental studies observed blood flow inside the aortic arch with rSCA cannulation and drew important conclusions. However, it is still unclear how blood streamlines into the arch. CFD may help improve the understanding of blood flow distribution. To date, some studies have reported on the hemodynamic analysis of cardiovascular diseases. We have even reported on the hemodynamic analysis of aortic arch pathologies by using CFD. ${ }^{8}$ Our previous study's findings showed that high wall shear stress or the oscillatory shear index may be associated with acute aortic dissection entry. We also reported on CFD analysis of the optimal design of the aortic arch reconstruction during the Norwood procedure. ${ }^{9}$ The anatomy of the aortic arch and design of the coronary artery bypass grafting could be evaluated by CFD. ${ }^{17}$ These studies have proven the availability of CFD. By using CFD, we can evaluate $3 \mathrm{D}$, patient-specific, time-resolved images of the blood streamline, velocity, wall shear stress, oscillatory shear index, and so forth. We think that CFD is the only method for evaluating blood flow inside the aortic arch with rSCA cannulation, because it is unrealistic to evaluate it by computed tomography or magnetic resonance imaging (MRI) while the patient is on cardiopulmonary bypass.

In the present study, we simulated rSCA and AscAo cannulation. Overall, we noticed that spiral flow was produced by the arterial cannula. Inside the dilated aorta, the blood streamline can spiral even without cardiopulmonary bypass. ${ }^{8}$ However, even inside a normal-sized aorta, the streamline spiraled with the arterial inflow. We hypothesize that atheromatous plaque may be dislodged because of this 
spiraled flow during cardiopulmonary bypass with both rSCA and AscAo cannulation. With 100\% flow, spiraled blood flow reached to the aortic root in both rSCA and AscAo simulation. This might cause dislodgement of plaque around the aortic root or AscAo.

Our results clarified that in all models with rSCA cannulation, the right common carotid artery was perfused by blood flow from the rSCA cannulation throughout the cardiac cycle in $50 \%$ and $75 \%$ flow. These findings were not observed in the AscAo cannulation model. Blood flow from the rSCA deflected blood flow from the AscAo and aortic arch toward the descending aorta. Although it is difficult to simulate the possibilities of cerebral embolization caused by aortic plaque without using particle tracking analysis, our streamline study results showed a cerebroprotective effect of rSCA cannulation. By deflecting the blood flow, the right common carotid artery was protected by the dislodged plaque at the AscAo.

During cardiopulmonary bypass, cerebral embolization occurs on the right side more frequently than on the left side. ${ }^{18}$ Therefore, protecting the right side of the supraaortic vessels is clinically important, and the effects of rSCA cannulation are significant.

In regard to the left side of the supra-aortic vessels, flow distribution was completely different from the right side and it depended on the aortic arch anatomy. In patients 1 and 2, flow distribution at the left common carotid artery was similar. In both patients, during the entire cardiac cycle, rSCA flow perfused the left common carotid artery. Therefore, mild dilatation of the AscAo $(45 \mathrm{~mm})$ did not affect the flow distribution of the left common carotid artery by rSCA cannulation. However, in patient 3, both left vessels were totally perfused by the native flow in systole. This was probably because the anatomic shape of the dilated distal arch influenced the blood flow distribution. We consider that the cerebroprotective effect on the left side was limited in the case with a distal arch aneurysm. In patient 4 with a bovine aortic arch, flow distribution of the left supra-aortic vessels was similar to that of patients' 1 and 2. Retrograde flow from the rSCA streamed into the aortic arch from the common trunk of the brachiocephalic artery and left common carotid artery. We expected that the left common carotid artery was perfused by flow from the rSCA flow, similar to the right common carotid artery, because of the common trunk. However, flow distribution of the left supra-aortic vessels was not different from that in patients 1 and 2 . The bovine arch did not play an important role in flow distribution with rSCA cannulation.

With AscAo cannulation, our analysis showed that a mixed flow streamline flowed into the 3 supra-aortic vessels in systole. In diastole, flow from the AscAo became dominant in all cases. Of note, in all models, the left common carotid artery was totally perfused by the flow from the AscAo cannula in diastole.

Flow velocity of supra-aortic vessel increased as the flow increased to $75 \%$ with rSCA cannulation. This was caused by increased blood flow to the arteries. The flow velocity was not as high as in the AscAo model compared with the rSCA model. This finding was previously reported by Minakawa and colleagues, ${ }^{16}$ who used a glass model simulation. They concluded that physicians should be cautious of the plaque location during rSCA cannulation, because dislodgement is possible. The clinical impacts of this increased velocity of the neck vessels are not yet clear. Further analysis of the flow velocity is required to determine the appropriate neck vessel flow during cardiopulmonary bypass.

\section{Study Limitations}

Although we established CFD models in reference to the results of 4-dimensional MRI, ${ }^{10}$ it is controversial whether calculated results, such as the flow velocity, are as accurate as hemodynamic parameters directly measured by a catheter or echocardiography. There are also limitations to the present study. First, to reduce the calculation cost, we used a fixed boundary condition, but wall motion with elastic properties should have been included to increase accuracy. However, estimation of the elastic vessel property distribution in atherosclerotic aortic disease is difficult; thus, a simple fluid-structure interaction calculation cannot solve this problem. Second, validation regarding the flow split to each branch or flow streamlines was insufficient. Even MRI flow measurements have limitations in spatial and temporal resolution; thus, there are technical limitations regarding the validation of CFD modeling. Third, we did not include vessel wall motion. Future studies should be performed on the basis of fluid-structure interaction modeling in terms of accurate elastic property and surrounding tissues from patient-specific images, ${ }^{19}$ and accurate boundary layer turbulence flow simulation. ${ }^{20}$ Fourth, idealized boundary conditions were used to compare the impact of each aortic arch pathology. Patients' specific cardiac function, aortic valve pathology, and hypertension were not considered in our calculations. Therefore, there may be a discrepancy between the individually measured data and calculated data. Fifth, the shape of the AscAo cannula is not ideal. We normally use a variety of shapes of arterial cannulas for AscAo cannulation. This time we simulated anastomosis of an $8-\mathrm{mm}$ graft to the AscAo, which may not be typical.

Finally, we analyzed blood flow on the basis of only the aortic lumen shape obtained from the enhanced computed tomography. For accurate embolic risk evaluation, particle-tracking analyses could be helpful. Although it is difficult to identify the plaque distribution and vulnerability to determine the starting point of the particle trajectories, 
there could be a reasonable stochastic approach. We might be able to hypothesize the aortic wall where the risk of plaque dislodgement was high as the starting point for the particle tracking. For evaluating atheromatous plaque dislodgement from the AscAo to the brain, particletracking analyses could simulate the movement of plaque realistically. We would like to perform a pathline analysis based on sufficient information of the aortic plaque distribution in a future study.

\section{CONCLUSIONS}

We can simulate blood flow inside the aortic arch by using rSCA cannulation as 3D, patient-specific, time-resolved images during 1 cardiac cycle. The right common carotid artery was perfused by blood flow from the rSCA cannula during $50 \%$ and $75 \%$ cardiopulmonary bypass flow. The left side of the supra-aortic branches was perfused by blood flow from the rSCA cannula and aortic valve. Anatomic characteristics may influence flow distribution of the neck vessels. A dilated AscAo and bovine aortic arch did not influence the flow distribution, but the distal arch aneurysm affected the left supra-aortic branch flow distribution. The clinical results of open surgery with rSCA cannulation at the Kyoto Prefectural University of Medicine were satisfactory with a low rate of neurologic deficits. Overall, rSCA cannulation may contribute to a cerebroprotective effect.

\section{Webcast}

You can watch a Webcast of this AATS meeting presentation by going to: http://webcast.aats.org/2016/Video/ Tuesday/05-17-16_Ballroom_I_1719_Numata-800.mp4.

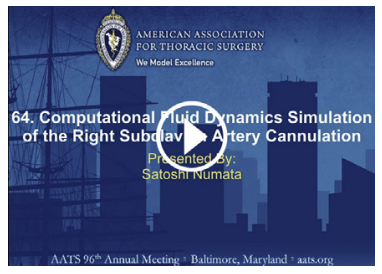

\section{Conflict of Interest Statement}

Authors have nothing to disclose with regard to commercial support.

\section{References}

1. Baribeau YR, Westbrook BM, Charlesworth DC, Maloney CT. Arterial inflow via an axillary artery graft for the severely atheromatous aorta. Ann Thorac Surg. 1998;66:33-7.

2. Sinclair MC, Singer RL, Manley NJ, Montesano RM. Cannulation of the axillary artery for cardiopulmonary bypass: safeguards and pitfalls. Ann Thorac Surg. 2003;75:931-4.

3. Numata S, Ogino H, Sasaki H, Hanafusa Y, Hirata M, Ando M, et al. Total arch replacement using antegrade selective cerebral perfusion with right axillary artery perfusion. Eur J Cardiothorac Surg. 2003;23:771-5.

4. Ogino H, Sasaki H, Minatoya K, Matsuda H, Tanaka H, Watanuki H, et al. Evolving arch surgery using integrated antegrade selective cerebral perfusion: impact of axillary artery perfusion. J Thorac Cardiovasc Surg. 2008;136:641-9.

5. Svensson LG, Blackstone EH, Rajeswaran J, Sabik JF III, Lytle BW, Gonzalez Stawinski G, et al. Does the arterial cannulation site for circulatory arrest influence stroke risk? Ann Thorac Surg. 2004;78:1274-84.

6. Sabik JF, Nemeh H, Lytle BW, Blackstone EH, Gillinov AM, Rajeswaran JM, et al. Cannulation of the axillary artery with a side graft reduces morbidity Ann Thorac Surg. 2004;77:1315-20.

7. Etz CD, Plestis KA, Kari FA, Silovitz D, Bodian CA, Spielvogel D, et al. Axillary cannulation significantly improves survival and neurologic outcome after atherosclerotic aneurysm repair of the aortic root and ascending aorta. Ann Thorac Surg. 2008;86:441-7.

8. Numata S, Itatani K, Kanda K, Doi K, Yamazaki S, Morimoto K, et al. Blood flow analysis of the aortic arch using computational fluid dynamics. Eur J Cardiothorac Surg. 2016;49:1578-85.

9. Itatani K, Miyaji K, Qian Y, Liu JL, Miyakoshi T, Murakami A, et al. Influence of surgical arch reconstruction methods on single ventricle workload in the Norwood procedure. J Thorac Cardiovasc Surg. 2011;144:130-8.

10. Itatani K. Advance in Hemodynamic Research. New York: Nova Science Publishers, Inc; 2015.

11. Hillebrand J, Konerding MA, Koch M, Kaufmann T, Steinseifer U, Moritz A, et al. Anatomic and flow dynamic considerations for safe right axillary artery cannulation. J Thorac Cardiovasc Surg. 2013;146:467-71.

12. Baek WK, Kim YS, Lee M, Yoon YH, Kim JT, Lim HK. Axillary artery cannulation in acute aortic dissection: a word of caution. Ann Thorac Surg. 2016;101 1573-4.

13. Battaloglu B, Secici S, Colak C, Disli OM, Erdil N, Kutlu R. Aberrant right sub clavian artery and axillary artery cannulation in type A aortic dissection repair Ann Thorac Surg. 2013;96:e1-2.

14. Maxwell BG, Harrington KB, Beygui RE, Oakes DA. Congenital anomalies of the aortic arch in acute type-a aortic dissection: implications for monitoring, perfusion strategy, and surgical repair. J Cardiothorac Vasc Anesth. 2014;28: 467-72.

15. Hedayati N, Sherwood JT, Schomisch SJ, Carino JL, Markowitz AH. Axillary artery cannulation for cardiopulmonary bypass reduces cerebral microemboli. $J$ Thorac Cardiovasc Surg. 2004;128:386-90.

16. Minakawa M, Fukuda I, Inamura T, Yamaoka H, Fukui K, Daitoku K, et al. Hydrodynamic evaluation of axillary artery perfusion for normal and diseased aorta. Gen Thorac Cardiovasc Surg. 2008;56:215-21.

17. Koyama S, Itatani K, Yamamoto T, Miyazaki S, Kitamura T, Taketani T, et al. Optimal bypass graft design for left anterior descending and diagonal territory in multivessel coronary disease. Interact Cardiovasc Thorac Surg. 2014;19:406-13.

18. Floyd TF, Shah PN, Price CC, Harris F, Ratcliffe SJ, Acker MA, et al. Clinically silent cerebral ischemic events after cardiac surgery: their incidence, regional vascular occurrence, and procedural dependence. Ann Thorac Surg. 2006;81: 2160-6.

19. Bertagna L, Elia MD, Perego M, Veneziani A. Data Assimilation in Cardiovascular Fluid-Structure Interaction Problems: An Introduction. Fluid-Structure Interaction and Biomedical Applications, Advances in Mathematical Fluid Me chanics. Basel: Springer; 2014

20. Takizawa K, Moorman C, Wright S, Purdue J, McPhail T, Chen PR, et al. Patientspecific arterial fluid-structure interaction modeling of cerebral aneurysms. Int $J$ Numer Meth Fluids. 2011;65:308-23.

Key Words: cardiopulonary bypass, fluid dynamics, subclavian artery

\section{Discussion}

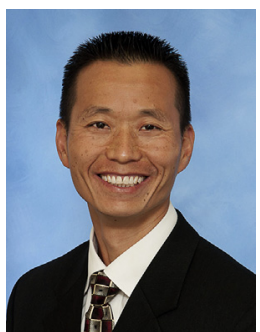

Dr Bo Yang (Ann Arbor, Mich). You choose $50 \%$ and $75 \%$ of flow for your modeling. Those are 2 snapshots. Why not choose different continuous change of the flow, such as $0 \mathrm{~L}, 1 \mathrm{~L}$, 2L, 3L, 4L, 5L, 6L? Then you will have a dose-response curve of distribution of the blood flow to the head vessels depending on flow rate, and your conclusion will be 
more convincing. When the flow is $100 \%$ (full cardiopulmonary bypass), there will be retrograde flow to the coronaries too. If the patient has calcification or plaque in the AscAo, there is a risk of increasing the coronary artery embolization. Your model with $100 \%$ flow will give us this additional important information.

Dr Satoshi Numata (Kyoto, Japan). If we do the analysis like that, we will be able to see more information about cannulation. But it takes time to complete the analysis, so for that reason we perform only the $50 \%$ and $75 \%$ flow. Now we are going to perform the $100 \%$ flow, so the no antegrade flow from ascending aortic valve simulation. We are going to perform that soon.

Dr Yang. We often use the innominate artery for arch aneurysm or acute type A aortic dissection. You chose the rSCA. What is the expectation of the flow distribution if the innominate artery is used for cannulation? Do you think the flow pattern will change? Do you think you will have more flow to the left common carotid artery and better prevention of the cerebral embolism?

Dr Numata. Are you using the brachiocephalic artery?

Dr Yang. The innominate artery.

Dr Numata. In our practice we always use the rSCA, because clamping the innominate artery is a bit risky for brain protection.

Dr Yang. We have not seen an increased risk of stroke. If you use the innominate artery, what do you expect?

Dr Numata. We haven't tried the simulation, but are you using the cannula or artificial graft?

Dr Yang. A Dacron graft sewn to the innominate artery.

Dr Numata. If you use grafts, the results probably are the same, I think, the flow from the innominate artery producing the retrograde flow into the AscAo. So the brain protection effect is probably the same.

Dr Yang. You might have more retrograde flow from the innominate artery into the arch. If I give your patient a computed tomography scan, can you use your model to tell me the best cannulation strategy? Can your model tell me the shear stress of the aortic wall? What is the risk the flow can dislodge the plaque of the aortic wall?

Dr Numata. That is a good point. If we insert the ascending aortic cannula, if we change the position, the flow pattern inside the aortic arch is different, and, as you said, the distribution of wall shear stress also is different.
We probably are able to perform the simulation of blood flow inside the aortic arch before surgery, and we can decide which cannulation site is the best for each patient.

Dr Saeed Ashraf (Wales, United Kingdom). I thoroughly enjoyed your research project. We have been doing this kind of work for the last couple of years. Just to continue from the previous speaker, we tried a different flow pattern and after $80 \%$ to $85 \%$ of flow, we were unable to see much of the retrograde or antegrade flow deflection. Did you see any difference between the size of the AscAo diameter and the flow pattern? This was an intriguing piece of work.

Dr Numata. According to our evaluation this time, the dilated AscAo may not influence the distribution of the flow to the neck vessels. We haven't evaluated the aortic dissection case yet, so we'll have to try that.

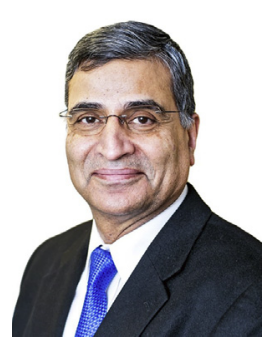

Dr Bansi Koul (Lund, Sweden). Thanks for sharing this fascinating information with us. I know that it is not part of your study, but have you studied how much blood flow was directed toward the right arm when you performed subclavian artery cannulation with a graft?

Dr Numata. In the clinical setting, we always monitor the right-hand pressure and the saturation of the brain, and it depends on the pressure and saturation findings. We snare the distal side of the subclavian artery to ensure enough flow to the arm and brain. In this simulation, I clamp the distal side of the subclavian artery for a comfortable calculation.



Dr Steven Lansman (Valhalla, $N Y$ ). Is there a quantitative way to evaluate these computational analyses or is one limited to a visual description of the color flow patterns?

Dr Estrera. Is there a way to quantify information?

Dr Numata. This is just a simulation, but we previously performed the normal, natural blood flow inside the aorta simulation previously. We also perform an MRI evaluation. We have a validation study comparing this simulation with the actual MRI study. So the MRI study is true. 